\title{
GPS Receiver Position Augmentation Using Correntropy Kalman Filter in Low Latitude Terrain
}

\author{
Sirish Kumar Pagoti ${ }^{1}$, Srilatha Indira Dutt Vemuri ${ }^{2}$, and Ganesh Laveti ${ }^{3}$ \\ ${ }^{1}$ Department of Electronics and Communication Engineering, Aditya Institute of Technology and \\ Management, India \\ ${ }^{2}$ Department of Electronics and Communication Engineering, GITAM University, India \\ ${ }^{3}$ Department of Electronics and Communication Engineering, GVP College of Engineering for Women, \\ India
}

\begin{abstract}
If any Global Positioning System (GPS) receiver is operated in low latitude regions or urban canyons, the visibility further reduces. These system constraints lead to many challenges in providing precise GPS position accuracy over the Indian subcontinent. As a result, the standalone GPS accuracy does not meet the aircraft landing requirements, such as Category I (CAT-I) Precision Approaches. However, the required accuracy can be achieved by augmenting the GPS. Among all these issues, the predominant factors that significantly influence the receiver position accuracy are selecting a user/receiver position estimation algorithm. In this article, a novel method is proposed based on correntropy and designated as Correntropy Kalman Filter (CKF) for precise GPS applications and GPS Aided Geosynchronous equatorial orbit Augmented Navigation (GAGAN) based aircraft landings over the low latitude Indian subcontinent. The real-world GPS data collected from a dual-frequency GPS receiver located in the southern region of the Indian subcontinent (IISc), Bangalore with Lat/Long: $13.021^{\circ} \mathrm{N} / 77.5^{\circ} \mathrm{E}$ ) is used for the performance evaluation of the proposed algorithm. Results prove that the proposed CKF algorithm exhibits significant improvement (up to 34\%) in position estimation compared to the traditional Kalman Filter.
\end{abstract}

Keywords: Accuracy, correntropy, correntropy kalman filter, global positioning system, kalman filter.

Received February 7, 2020; accepted February 7, 2021

https://doi.org/10.34028/iajit/19/1/9

\section{Introduction}

The generic term preferred for the satellite-based navigation system is the Global Navigation Satellite System (GNSS), which covers global satellite constellation such as Global Positioning System (GPS), Global Orbiting Navigation Satellite System (GLONASS), BeiDou, Galileo, etc., Currently, GPS is the only full-fledged global satellite constellation system in GNSS; it has 32 satellites more significant than the nominal figure of 24 satellites. All over this globe, a minimum of 14 to 18 satellite signal systems are available andoperating fully for various sources since 1995 in both civilian and military fields. For continuous worldwide coverage, the arrangement of GPS satellites is such that four satellites are arranged in each of 6 orbits [19].

The estimation problem has been a significant issue in industrial application and research areas covering the processing of signals, optimization, and navigational decisions; many marked fields requiring estimation, identification of system, tracking of the target, and localization. In linear dynamics and systematic applications, Kalman Filter (KF) is used to solve estimation. In general, KF and its modifications
$[12,13,15,20,23]$ have excellent performance in Gaussian variety noises. However, their operations get degraded when non-Gaussian situations are predominantly in a system with disturbance of impulsive noises. Aforementioned, KF and Extended Kalman Filter (EKF) $[12,13,20]$ are not suitable for systems that are disturbed by heavy-tailed impulsive noises. Thus, modifications in the KF are necessary to overcome this difficulty. In this paper, KF has been modified based on the correntropy criterion [17] to improve the accuracy of GPS receiver position in low latitude regions like India. The proposed Correntropy Kalman Filter (CKF) adopts the robust correntropy criterion as the optimality criterion instead of using the well-known Minimum Mean Square Error (MMSE). Unless mentioned in this article, correntropy of error may be utilized as a cost function for adaptive training of the system. It perceives that correntropy, having the advantage of being local, can be useful for the situations in which the measurement noise has a nonzero mean, non-Gaussian pattern with large outliers. Like the traditional KF, the state mean vector and covariance matrix propagation equations give prior estimations of the state and covariance matrix in CKF. A novel Fixed-point algorithm is then used to update 
the posterior estimates. The proposed algorithm results are then compared with the traditional KF algorithm.

The paper's structure is as follows. In section 2, a short review of the literature and motivation in this area of research is presented. Section 3 covers the correntropy notation and basics of KF estimator; CKF implementation and essential steps to estimate accurate user/receiver position are discussed in section 4 . In section 5, elaborated the results on estimated position and errors of GPS receiver in $\mathrm{X}, \mathrm{Y}$, and $\mathrm{Z}$ direction, comparison of the proposed method CKF with the traditional KF estimator are presented, and numerous statistical factors (2-D and 3-D) are tabulated. The overall conclusions, along with the suggestions for possible future extensions, are provided in section 6 . Finally, in Table 6 of the conclusions, the nomenclature used in explaining the algorithms in a deterministic manner is presented.

\section{Review of Literature}

Precise positioning and robust surveillance systems are crucial to secure countries such as India, with a long geographical frontier and an expansive coastline. This has led to the development and advancement of many of these systems. Aviation, maritime, mining, military, medicine, and agriculture are some of these system's real-world applications. Global positioning of an unknown object also plays a prominent role in the civil aviation and defense sector fields for aircraft landings, navigation of ships, etc. Literature also provides an enormous number of critical applications of positioning systems and shows the increase in need and demand for positioning systems $[1,18]$. The different positioning algorithm strategies to solve these measurement equations like linear, nonlinear, and iterative, closed-form solutions are also studied and analyzed $[10,16,24]$.

To improve the accuracy, Firefly Algorithm (FA) and Teaching Learning Based Optimization (TLBO) algorithms were developed [4]. Estimation accuracy can be further enhanced by using KF, EKF is also studied [7, 9, 12], which can estimate the required state under noisy conditions. Later, a new filter of Kalman family (weighted Kalman Filter) was developed based on the variance estimation method [22]. This method improves accuracy by up to $30 \%$ and Central Processing Unit (CPU) time up to $80 \%$ in speed and velocity. A new filtering algorithm Maximum Correntropy Kalman Filter (MCKF) [5] like the Kalman type has recently been introduced. It depends primarily on the criterion of correntropy [17], which is an alternative to the renowned measure of MMSE [3, 6]. This filtering algorithm makes the traditional Kalman filter more effective and robust. Because state and covariance matrix prior and posterior estimates are the same as KF, this algorithm was best suited for systems influenced by noisy environments (Gaussian,
non-Gaussian, and impulsive) $[8,11,14,21]$. In the present paper, to address the problem of accuracy improvement in low latitude regions like India, a new method in the implementation of KF based on the correntropy criterion designated as CKF, is proposed. The work presented in this paper is aimed at strengthening the proposed GNSS/GPS based navigation system.

The proposed algorithm CKF used in this paper is useful for navigation and surveying in urban canyons and dense forest areas where the satellite visibility is low. In this paper, the proposed CKF performance is evaluated with real-time pseudo-range GPS data and can be implemented straight away to carrier phase measurement-based critical GPS applications like surveying and farming. The iterative algorithm implemented for receiver position estimation can also solve various other optimization problems encountered in the control and automation industry. They are also useful for Geographic Information System (GIS) and accurate aerial mapping applications.

Literature survey unveils that to increase demand for an accurate positioning system, optimal measurement strategies and precise navigation solutions are necessary. For critical GPS applications like Category I (CAT-I) aircraft landings, low variance in estimation is crucial besides the estimated position accuracy. Moreover, the number of onboard system parameters and dynamics needs to be analyzed while estimating the position. This section summarizes the prior and conventional work is done to consider the literature related to the research.

\section{Correntropy Notation}

Correntropy [17] is a nonlinear yardstick of resemblance between a couple of arbitrary factors $A, B$ with joint probability function $P_{A \mid B}(a \mid b)$.

correntropy is characterized by

$$
\mathrm{R}(\mathrm{A} \mid \mathrm{B})=\mathrm{E}\left[\mathrm{k}_{\sigma}(\mathrm{A} \mid \mathrm{B})\right]=\int \mathrm{k}_{\sigma}(\mathrm{a} \mid \mathrm{b}) \mathrm{CP}_{\mathrm{A} \mid \mathrm{B}}(\mathrm{a} \mid \mathrm{b})
$$

Where $E$ is the expectation operator, $k_{\sigma}(\cdot, \cdot)$ is the definite kernel of any continuous variable.

The gaussian kernel $\left(J_{\sigma}\right)[12]$ is used in our framework.

Consequently, Equation (1) becomes

$$
\mathrm{k}_{\sigma}(\mathrm{a} \mid \mathrm{b})=\mathrm{J}_{\sigma}(\mathrm{e})=\exp \left(-\frac{\mathrm{e}^{2}}{2 \sigma^{2}}\right)
$$

Where ' $e$ ' is the error, which is defined as the contrast of the expected output to the actual output and ' $\sigma$ ' stands for the width of the kernel.

The joint PDF of $A$ and $B$ is generally unknown in practice, and there are only a limited number of samples available.

In such scenarios, a sample mean estimator can be used to estimate the correntropy:

$$
\widehat{\mathrm{R}}(\mathrm{A} \mid \mathrm{B})=\frac{1}{\mathrm{M}} \sum_{\mathrm{k}=1}^{\mathrm{m}} \mathrm{J}_{\sigma}(\mathrm{e}(\mathrm{k}))
$$


Where, $e(k)=a(k)-b(k)$ with $\{a(k), b(k)\}_{k=1}^{M}$ being $M$ samples drawn from $P_{A \mid B}(a \mid b)$

Now we have been expanding the Taylor series of the gaussian kernel

$$
R(A \mid B)=\sum_{i=0}^{\infty} \frac{(-1)^{i}}{2^{i} \sigma^{2 i} i !} E\left[(A-B)^{2 i}\right]
$$

As shown, "the correntropy is a weighted sum of the random variable's $(A-B)$ even order moments. The kernel bandwidth is a weighting parameter for $2^{\text {nd }}$ and higher-order moments." The correntropy is dominated by $2^{\text {nd }}$ order moments when the $\sigma$ is much greater than the results dynamic range. Correntropy depends on the size of the kernel that needs to be chosen as per the application. Although the kernel size is measured as a percentage, its net impact varies due to the predicted value operation in the concept's correntropy. It can be proved that correntropy approaches correlation for the kernel's sizes, which is larger than recommended. The kernel size can regulate the usage of higher-order moment information for correlating; this is a beautiful and unique concept with proper selection; the outcomes for correntropy are equivalent to robust statistical approaches. Furthermore, due to the smooth dependency of correntropy in kernel size, the kernel size output's sensitivity must be much smaller than the choice of thresholds. However, kernel size estimation methods must be deployed appropriately for practical applications.

\subsection{Kalman Filter}

KF offers a useful tool for dealing with linear system state estimation, an efficient estimator under linear and Gaussian assumptions. This filter mainly consists of three factors: predicting, observing, and estimating [9, 20]. Considering a linear system described with the resulting equations of state and measurement:

$$
\left.\begin{array}{c}
\mathrm{a}(\underline{\mathrm{t}})=\mathrm{Z}(\underline{\mathrm{t}}-1) \mathrm{a}(\underline{\mathrm{t}}-1)+\mathrm{x}(\underline{\mathrm{t}}-1) \\
\mathrm{b}(\underline{\mathrm{t}})=\mathrm{S}(\underline{\mathrm{t}}) \mathrm{a}(\underline{\mathrm{t}})+\mathrm{y}(\underline{\mathrm{t}}) \\
\mathrm{E}\left[\mathrm{x}(\underline{\mathrm{t}}-1) \mathrm{x}^{\mathrm{T}}(\underline{\mathrm{t}}-1)\right]=\mathrm{X}(\underline{\mathrm{t}}-1) \\
\mathrm{E}\left[\mathrm{y}(\underline{\mathrm{t}}) \mathrm{y}^{\mathrm{T}}(\underline{\mathrm{t}})\right]=\mathrm{Y}(\underline{\mathrm{t}})
\end{array}\right\}
$$

Kalman Filter has, in general, two steps: Predict and Update.

\section{- Predict:}

The predicted mean and covariance matrix are given by

$$
\begin{gathered}
\hat{\mathrm{a}}(\underline{\mathrm{t}} \mid \underline{\mathrm{t}}-1)=\mathrm{Z}(\underline{\mathrm{t}}-1) \hat{\mathrm{a}}(\underline{\mathrm{t}}-1 \mid \underline{\mathrm{t}}-1) \\
\mathrm{Q}(\underline{\mathrm{t}} \mid \underline{\mathrm{t}}-1)=\mathrm{Z}(\underline{\mathrm{t}}-1) \mathrm{Q}(\underline{\mathrm{t}}-1 \mid \underline{\mathrm{t}}-1) \mathrm{Z}^{\mathrm{T}}(\underline{\mathrm{t}}-1)+\mathrm{X}(\underline{\mathrm{t}}-1)
\end{gathered}
$$

- Update:

The gain of the KF is computed as

$$
\mathrm{T}(\underline{\mathrm{t}})=\mathrm{Q}(\underline{\mathrm{t}} \underline{\mathrm{t}}-\underline{1}) \mathrm{S}^{\mathrm{T}}(\underline{\mathrm{t}})\left(\mathrm{S}(\underline{\mathrm{t}}) \mathrm{Q}(\underline{\mathrm{t}} \mathrm{t} \underline{\mathrm{t}}-1) S^{\mathrm{T}}(\underline{\mathrm{t}})+\mathrm{Y}(\underline{\mathrm{t}})\right)^{-1}
$$

It is observed that the posterior state is the prior state coupled with innovation weighted by the KF gain,

$$
\hat{a}(\underline{t} \mid \underline{t})=\hat{a}(\underline{t} \mid \underline{t}-1)+T(\underline{t})(b(\underline{t})-S(\underline{t}) \hat{a}(\underline{t} \mid \underline{t}-1))
$$

Further, the posterior covariance is updated recursively:

$$
Q(\underline{t} \mid \underline{t})=(I-T(\underline{t}) S(\underline{t})) Q(\underline{t} \mid \underline{t}-1)(I T(\underline{t}) S(\underline{t}))^{T}+T(\underline{t}) Y(\underline{t}) T^{T}(\underline{t})
$$

\section{Correntropy Kalman Filter}

Conventional KF deteriorates performance in nonGaussian noises [10,17], particularly for the system is distributed by impulsive noises. As discussed above, $\mathrm{KF}$ is formed on the MMSE criterion [6], which is the leading cause of the problem. It collects only secondorder error statistics and becomes susceptible to large outliers. Using the correntropy criterion, CKF is derived to solve it. This filter works best in nonGaussian environments [3] as correntropy has second and higher-order error moments. The CKF algorithm's computational flow chart is presented in Figure 1.

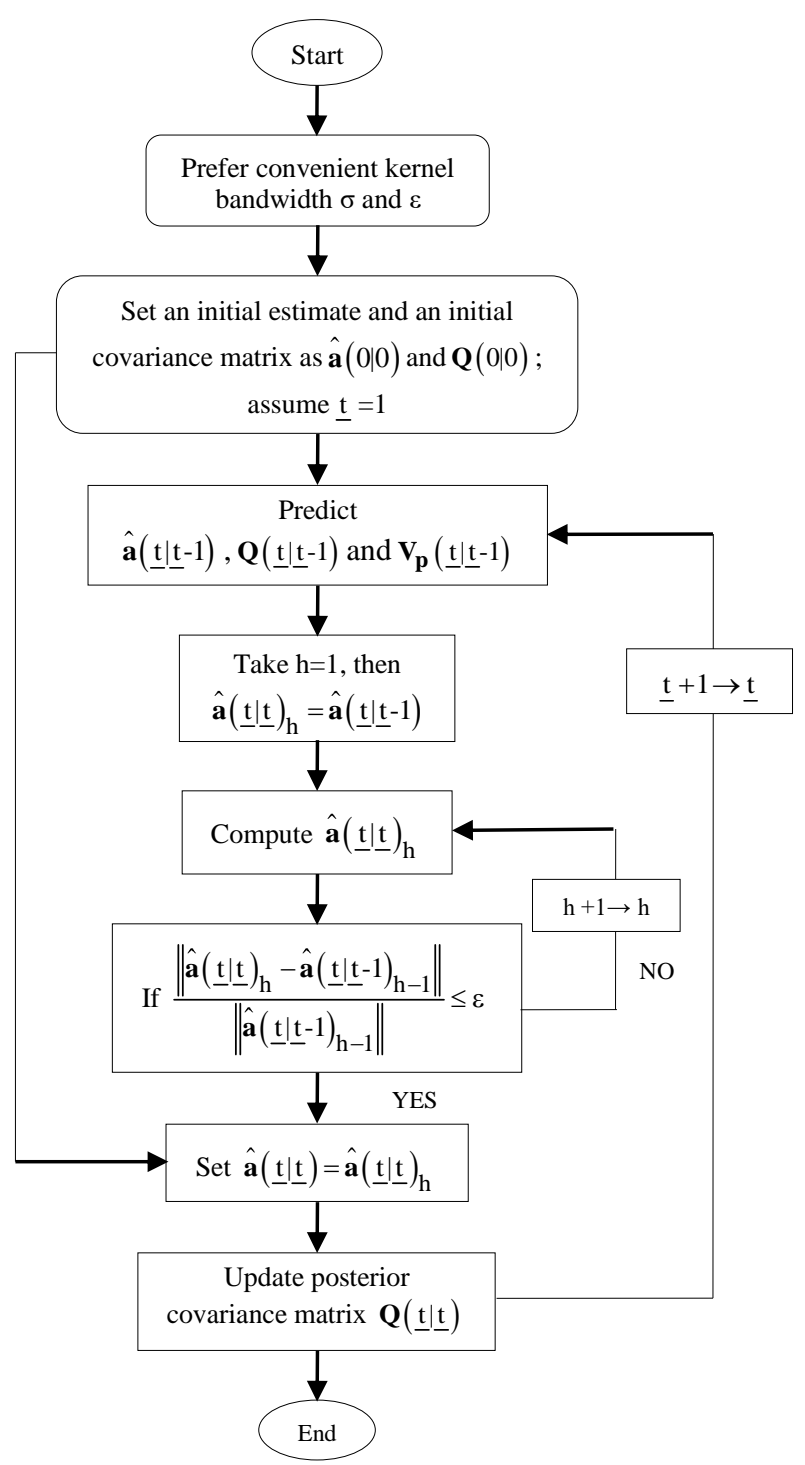

Figure 1. Computational flow chart of correntropy kalman filter.

We have the linear model mentioned in the last section 


$$
\left[\begin{array}{c}
\hat{a}(\underline{\mathrm{t}} \mid \underline{\mathrm{t}}-1) \\
\mathrm{b}(\underline{\mathrm{t}})
\end{array}\right]=\left[\begin{array}{c}
\mathrm{I} \\
\mathrm{S}(\underline{\mathrm{t}})
\end{array}\right] \mathrm{a}(\underline{\mathrm{t}})+\mathrm{r}(\underline{\mathrm{t}})
$$

Where, $I$ is identity matrix, and $r(\underline{t})$ is

$$
\mathrm{r}(\underline{\mathrm{t}})=\left[\begin{array}{c}
-(\mathrm{a}(\underline{\mathrm{t}})-\hat{\mathrm{a}}(\underline{\mathrm{t}} \mid \underline{\mathrm{t}}-1)) \\
\mathrm{y}(\mathrm{t})
\end{array}\right]
$$

With,

$$
\begin{gathered}
\mathrm{E}\left[\mathrm{r}(\underline{\mathrm{t}}) \mathrm{r}^{\mathrm{T}}(\underline{\mathrm{t}})\right]=\left[\begin{array}{cc}
\mathrm{Q}(\underline{\mathrm{t}} \mathrm{t}-\underline{\mathrm{t}}-1) & 0 \\
0 & \mathrm{Y}(\underline{\mathrm{t}})
\end{array}\right] \\
=\left[\begin{array}{cc}
\mathrm{V}_{\mathrm{p}}(\underline{\mathrm{t}} \mid \underline{\mathrm{t}}-1) \mathrm{V}_{\mathrm{p}}^{\mathrm{T}}(\underline{\mathrm{t}} \mid \underline{\mathrm{t}}-1) & 0 \\
0 & \mathrm{~V}_{\mathrm{r}}(\underline{\mathrm{t}}) \mathrm{V}_{\mathrm{r}}^{\mathrm{T}}(\underline{\mathrm{t}})
\end{array}\right] \\
=\mathrm{V}(\underline{\mathrm{t}}) \mathrm{V}^{\mathrm{T}}(\underline{\mathrm{t}})
\end{gathered}
$$

Where, $V(\underline{t})$ is determined by cholesky factorization of $E\left[r(t) r^{T}(t)\right]$.

Multiplication of both sides of Equation (13) by $V^{-1}(\underline{t})$,

$$
\mathrm{C}(\underline{\mathrm{t}})=\mathrm{U}(\underline{\mathrm{t}}) \mathrm{a}(\underline{\mathrm{t}})+\mathrm{e}(\underline{\mathrm{t}})
$$

Where,

$$
\begin{gathered}
\mathrm{C}(\underline{\mathrm{t}})=\mathrm{V}^{-1}(\mathrm{t})\left[\begin{array}{c}
\hat{\mathrm{a}}(\underline{\mathrm{t}} \mid \underline{\mathrm{t}}-1) \\
\mathrm{b}(\underline{\mathrm{t}})
\end{array}\right], \\
\mathrm{U}(\underline{\mathrm{t}})=\mathrm{V}^{-1}(\underline{\mathrm{t}})\left[\begin{array}{c}
\mathrm{I} \\
\mathrm{S}(\underline{\mathrm{t}})
\end{array}\right], \mathrm{e}(\underline{\mathrm{t}})=\mathrm{V}^{-1}(\underline{\mathrm{t}}) \mathrm{r}(\underline{\mathrm{t}})
\end{gathered}
$$

Since $E\left[e(\underline{t}) e^{T}(\underline{t})\right]=I$

Here, $e(t)$ is a white residual error.

Now, the following cost function based on correntropy is proposed:

$$
\mathrm{G}_{\mathrm{N}}(\mathrm{a}(\underline{\mathrm{t}}))=\frac{1}{\mathrm{~N}} \sum_{\mathrm{k}=1}^{\mathrm{N}} \mathrm{J}_{\sigma}\left(\mathrm{c}_{\mathrm{k}}(\underline{\mathrm{t}})-\mathrm{u}_{\mathrm{k}}(\underline{\mathrm{t}}) \mathrm{a}(\underline{\mathrm{t}})\right)
$$

Where $c_{k}(\underline{t})$ is the $\mathrm{k}^{\text {th }}$ element of $C(\underline{t}), u_{k}(\underline{t})$ is the $\mathrm{k}^{\text {th }}$ row of $U(\underline{t})$, and $\mathrm{N}=\mathrm{i}+\mathrm{j}$ is the dimension of $C(\underline{t})$.

Under correntropy criterion, optimal estimate for $a(\underline{t})$ is given by

$$
\hat{\mathrm{a}}(\underline{\mathrm{t}})=\operatorname{argmax}_{\mathrm{a}(\mathrm{t})} \mathrm{G}_{\mathrm{N}}(\mathrm{a}(\underline{\mathrm{t}}))=\operatorname{argmax}_{\mathrm{a}(\mathrm{t})} \sum_{\mathrm{k}=1}^{\mathrm{N}} \mathrm{J}_{\sigma}\left(\mathrm{e}_{\mathrm{k}}(\underline{\mathrm{t}})\right)
$$

Where $e_{k}(\underline{t})$ is the $\mathrm{k}^{\text {th }}$ element of $e(t)$ :

$$
\mathrm{e}_{\mathrm{k}}(\underline{\mathrm{t}})=\mathrm{c}_{\mathrm{k}}(\underline{\mathrm{t}})-\mathrm{u}_{\mathrm{k}}(\underline{\mathrm{t}}) \mathrm{a}(\underline{\mathrm{t}})
$$

It allows the optimal solution by solving

$$
\frac{\partial \mathrm{G}_{\mathrm{N}}(\mathrm{a}(\mathrm{t}))}{\partial \mathrm{a}(\mathrm{t})}=\sum_{\mathrm{k}=1}^{\mathrm{N}}\left[\mathrm{J}_{\sigma}\left(\mathrm{e}_{\mathrm{k}}(\underline{\mathrm{t}})\right) \mathrm{u}_{\mathrm{k}}^{\mathrm{T}}(\underline{\mathrm{t}})\left(\mathrm{c}_{\mathrm{k}}(\underline{\mathrm{t}})-\mathrm{u}_{\mathrm{k}}(\underline{\mathrm{t}}) \mathrm{a}(\underline{\mathrm{t}})\right)\right]=0
$$

It quickly follows

$$
\mathrm{a}(\underline{\mathrm{t}})=\left(\sum_{\mathrm{k}=1}^{\mathrm{N}}\left[\mathrm{J}_{\sigma}\left(\mathrm{e}_{\mathrm{k}}(\underline{\mathrm{t}})\right) \mathrm{u}_{\mathrm{k}}^{\mathrm{T}}(\underline{\mathrm{t}}) \mathrm{u}_{\mathrm{k}}(\mathrm{t})\right]\right)^{-1} \times\left(\sum_{\mathrm{k}=1}^{\mathrm{N}}\left[\mathrm{J}_{\sigma}\left(\mathrm{e}_{\mathrm{k}}(\mathrm{t})\right) \mathrm{u}_{\mathrm{k}}^{\mathrm{T}}(\underline{\mathrm{t}}) \mathrm{c}_{\mathrm{k}}(\mathrm{t})\right]\right)
$$

Since $e_{k}(\underline{t})=c_{k}(\underline{t})-u_{k}(\underline{t}) a(\underline{t})$

In addition, the optimal solution of Equation (20) is a fixed point equation $a(t)$ [2] and can be rephrased as

$$
\mathrm{a}(\underline{\mathrm{t}})=\mathrm{f}(\mathrm{a}(\underline{\mathrm{t}}))
$$

With

$$
\begin{gathered}
\mathrm{f}(\mathrm{a}(\underline{\mathrm{t}}))=\left(\sum_{\mathrm{k}=1}^{\mathrm{N}}\left[\mathrm{J}_{\sigma}\left(\mathrm{c}_{\mathrm{k}}(\underline{\mathrm{t}})-\mathrm{u}_{\mathrm{k}}(\underline{\mathrm{t}}) \mathrm{a}(\underline{\mathrm{t}})\right) \mathrm{u}_{\mathrm{k}}^{\mathrm{T}}(\underline{\mathrm{t}}) \mathrm{u}_{\mathrm{k}}(\underline{\mathrm{t}})\right]\right)^{-1} \\
\times\left(\sum_{\mathrm{k}=1}^{\mathrm{N}}\left[\mathrm{J}_{\sigma}\left(\mathrm{c}_{\mathrm{k}}(\underline{\mathrm{t}})-\mathrm{u}_{\mathrm{k}}(\underline{\mathrm{t}}) \mathrm{a}(\underline{\mathrm{t}})\right) \mathrm{u}_{\mathrm{k}}^{\mathrm{T}}(\underline{\mathrm{t}}) \mathrm{c}_{\mathrm{k}}(\underline{\mathrm{t}})\right]\right)
\end{gathered}
$$

We can easily obtain a fixed point iterative algorithm as

$$
\hat{\mathrm{a}}(\mathrm{t})_{\mathrm{h}+1}=\mathrm{f}\left(\hat{\mathrm{a}}(\underline{\mathrm{t}})_{\mathrm{h}}\right)
$$

Where $\hat{a}(\underline{t})_{h}$ represents the required solution at the fixed point iteration ' $h$ '.

The equation of the fixed point i.e., Equation (20) can be also expressed as

$$
\mathrm{a}(\underline{\mathrm{t}})=\left(\mathrm{U}^{\mathrm{T}}(\underline{\mathrm{t}}) \mathrm{H}(\underline{\mathrm{t}}) \mathrm{U}(\underline{\mathrm{t}})\right)^{-1} \mathrm{U}^{\mathrm{T}}(\underline{\mathrm{t}}) \mathrm{H}(\underline{\mathrm{t}}) \mathrm{C}(\underline{\mathrm{t}})
$$

Where, $\mathrm{H}(\underline{\mathrm{t}})=\left[\begin{array}{cc}\mathrm{H}_{\mathrm{a}}(\mathrm{t}) & 0 \\ 0 & \mathrm{H}_{\mathrm{b}}(\mathrm{t})\end{array}\right]$

With,

$$
\begin{gathered}
\mathrm{H}_{\mathrm{a}}(\underline{\mathrm{t}})=\operatorname{diag}\left(\mathrm{J}_{\sigma}\left(\mathrm{e}_{1}(\underline{\mathrm{t}})\right), \ldots \ldots \ldots \mathrm{J}_{\sigma}\left(\mathrm{e}_{\mathrm{i}}(\underline{\mathrm{t}})\right)\right) \\
\mathrm{H}_{\mathrm{b}}(\underline{\mathrm{t}})=\operatorname{diag}\left(\mathrm{J}_{\sigma}\left(\mathrm{e}_{\mathrm{i}+1}(\underline{\mathrm{t}})\right), \ldots \ldots \ldots \mathrm{J}_{\sigma}\left(\mathrm{e}_{\mathrm{i}+\mathrm{j}}(\underline{\mathrm{t}})\right)\right)
\end{gathered}
$$

Equation (23) can be further rewritten as per the following:

$$
\mathrm{a}(\underline{\mathrm{t}})=\hat{\mathrm{a}}(\underline{\mathrm{t}} \mid \underline{\mathrm{t}}-1)+\overline{\mathrm{T}}(\underline{\mathrm{t}})(\mathrm{b}(\underline{\mathrm{t}})-\mathrm{S}(\underline{\mathrm{t}}) \hat{\mathrm{a}}(\underline{\mathrm{t}} \mid \underline{\mathrm{t}}-1))
$$

Where,

$$
\left\{\begin{array}{c}
\overline{\mathrm{T}}(\underline{\mathrm{t}})=\overline{\mathrm{Q}}(\underline{\mathrm{t}} \mid \underline{\mathrm{t}}-1) S^{\mathrm{T}}(\underline{\mathrm{t}})\left(\mathrm{S}(\underline{\mathrm{t}}) \overline{\mathrm{Q}}(\underline{\mathrm{t}} \mid \underline{\mathrm{t}}-1) S^{\mathrm{T}}(\underline{\mathrm{t}})+\overline{\mathrm{Y}}(\mathrm{t})\right)^{-1} \\
\overline{\mathrm{Q}}(\underline{\mathrm{t}} \mid \underline{\mathrm{t}}-1)=\mathrm{V}_{\mathrm{p}}(\underline{\mathrm{t}} \mid \underline{\mathrm{t}}-1) \mathrm{H}_{\mathrm{a}}^{-1}(\underline{\mathrm{t}}) \mathrm{V}_{\mathrm{p}}^{\mathrm{T}}(\underline{\mathrm{t}} \mid \underline{\mathrm{t}}-1) \\
\overline{\mathrm{Y}}(\underline{\mathrm{t}})=\mathrm{V}_{\mathrm{r}}(\underline{\mathrm{t}}) \mathrm{H}_{\mathrm{b}}^{-1}(\underline{\mathrm{t}}) \mathrm{V}_{\mathrm{r}}^{\mathrm{T}}(\mathrm{t})
\end{array}\right.
$$

\subsection{Steps in Computing Correntropy Kalman Filter}

Equation (24) is a fixed-point type for $a(\underline{t})$ as $\bar{T}(\underline{t})$ is dependent on $\bar{Q}(\underline{t} \mid \underline{t}-1)$ and $\bar{Y}(\underline{t})$, which are related to $a(\underline{t})$ via $H_{a}(\underline{t})$ and $H_{b}(\underline{t})$. Hence further, the optimal solution of Equation (24) also depends on the prior estimate $\widehat{a}(\underline{t} \mid \underline{t}-1)$, which can be computed by Equation (8) utilizing the latest estimate $\widehat{a}(\underline{t}-1 \mid \underline{t}-1)$. From the above assumptions, the proposed CKF algorithm can be summed up as follows:

- Step 1: Assume that $\underline{t}=1$; Pick the right kernel bandwidth $\sigma$ and a positive small number $\varepsilon$;

Set an initial estimate and an initial covariance matrix as $\hat{a}(0 \mid 0), \mathrm{Q}(0 \mid 0)$ respectively;

- Step 2: Equations (8) and (9) are used to obtain $\hat{\mathrm{a}}(\underline{\mathrm{t}} \mid \underline{\mathrm{t}}-1)$ and $Q(\underline{t} \mid \underline{t}-1)$ and use the factorization of Cholesky to get the $V_{p}(\underline{t} \mid \underline{t}-1)$;

- Step 3: Assume that $h=1$ and $\widehat{a}(\underline{t} \mid \underline{t})_{h}=\hat{a}(\underline{t} \mid \underline{t}-1)$, where $\hat{a}(\underline{t} \mid \underline{t})_{h}$ represents the estimated state at the fixed-point iteration $h$;

- Step 4: Use Equations (26)-(32) to calculate $\hat{a}(\underline{t} \mid \underline{t})_{\mathrm{h}}$

$$
\hat{\mathrm{a}}(\underline{\mathrm{t}} \mid \mathrm{t})_{\mathrm{h}}=\hat{\mathrm{a}}(\underline{\mathrm{t}} \mid \underline{\mathrm{t}}-1)+\overline{\mathrm{T}}(\underline{\mathrm{t}})(\mathrm{b}(\underline{\mathrm{t}})-\mathrm{S}(\underline{\mathrm{t}}) \hat{\mathrm{a}}(\underline{\mathrm{t}} \mid \underline{\mathrm{t}}-1))
$$

With $\overline{\mathrm{T}}(\underline{\mathrm{t}})=$

$$
\overline{\mathrm{Q}}(\underline{\mathrm{t}} \mid \underline{\mathrm{t}}-1) S^{\mathrm{T}}(\underline{\mathrm{t}})\left(\mathrm{S}(\underline{\mathrm{t}}) \overline{\mathrm{Q}}(\underline{\mathrm{t}} \mid \underline{\mathrm{t}}-1) \mathrm{S}^{\mathrm{T}}(\underline{\mathrm{t}})+\overline{\mathrm{Y}}(\mathrm{t})\right)^{-1}
$$




$$
\begin{gathered}
\overline{\mathrm{Q}}(\underline{\mathrm{t}} \mid \underline{\mathrm{t}}-1)=\mathrm{V}_{\mathrm{p}}(\mathrm{t} \mid \underline{\mathrm{t}}-1) \overline{\mathrm{H}}_{\mathrm{a}}^{-1}(\underline{\mathrm{t}}) V_{\mathrm{p}}^{\mathrm{T}}(\underline{\mathrm{t}} \mid \underline{\mathrm{t}}-1) \\
\overline{\mathrm{Y}}(\underline{\mathrm{t}})=\mathrm{V}_{\mathrm{r}}(\underline{\mathrm{t}}) \overline{\mathrm{H}}_{\mathrm{b}}^{-1}(\underline{\mathrm{t}}) \mathrm{V}_{\mathrm{r}}^{\mathrm{T}}(\underline{\mathrm{t}}) \\
\overline{\mathrm{H}}_{\mathrm{a}}(\underline{\mathrm{t}})=\operatorname{diag}\left(\mathrm{J}_{\sigma}\left(\overline{\mathrm{e}}_{1}(\underline{\mathrm{t}})\right), \ldots \ldots \ldots \mathrm{J}_{\sigma}\left(\overline{\mathrm{e}}_{\mathrm{i}}(\underline{\mathrm{t}})\right)\right) \\
\overline{\mathrm{H}}_{\mathrm{b}}(\underline{\mathrm{t}})=\operatorname{diag}\left(\mathrm{J}_{\sigma}\left(\overline{\mathrm{e}}_{\mathrm{i}+1}(\underline{\mathrm{t}})\right), \ldots \ldots \ldots \mathrm{J}_{\sigma}\left(\overline{\mathrm{e}}_{\mathrm{i}+\mathrm{j}}(\underline{\mathrm{t}})\right)\right) \\
\left.\overline{\mathrm{e}}_{\mathrm{k}}(\underline{\mathrm{t}})=\mathrm{c}_{\mathrm{k}}(\underline{\mathrm{t}})-\mathrm{u}_{\mathrm{k}}(\underline{\mathrm{t}}) \hat{\mathrm{a}} \mathrm{a} \mathrm{t} \mid \mathrm{t}\right)_{\mathrm{h}-1}
\end{gathered}
$$

- Step 5: The estimation of the current step and the last step is compared. For Equation (33) to be valid, set $\hat{\mathrm{a}}(\underline{\mathrm{t}} \mid \underline{\mathrm{t}})=\hat{\mathrm{a}}(\underline{\mathrm{t}} \mid \underline{\mathrm{t}})_{\mathrm{h}}$ and proceed to step (6). Otherwise, $\mathrm{h}+1 \rightarrow \mathrm{h}$ and go back to step (4).

$$
\frac{\| \hat{\mathrm{a}}(\underline{\mathrm{t}} \mid \mathrm{t}))_{\mathrm{h}}-\hat{\mathrm{a}}(\mathbf{t} \mid \mathrm{t})_{\mathrm{h}-1} \|}{\left\|\hat{\mathrm{a}} \mathrm{t}(\mathrm{t})_{\mathrm{h}-1}\right\|} \leq \varepsilon
$$

- Step 6: The posterior covariance matrix is updated by Equation (34), $\underline{\mathrm{t}}+1 \rightarrow \underline{\mathrm{t}}$, and go back to step (2).

$$
\left.\mathrm{Q}(\mathrm{t}|\mathrm{t}| \mathrm{t})=(\mathrm{I}-\overline{\mathrm{T}}(\mathrm{t}) S(\mathrm{t})) \mathrm{Q}(\mathrm{t} \mid \underline{t}-1)(\mathrm{I}-\overline{\mathrm{T}}(\mathrm{t}) \mathrm{S}(\mathrm{t}))^{\mathrm{T}}+\overline{\mathrm{T}}(\underline{\mathrm{t}}) \mathrm{Y}(\mathrm{t})\right)^{\mathrm{T}}(\underline{\mathrm{t}})
$$

The little positive value $\varepsilon$ for the fixed point iteration provides a stop condition. A key parameter in CKF is the kernel bandwidth $\sigma$. A smaller bandwidth in the kernel usually improves the performance but makes the algorithm converge slower. In comparison, as $\sigma$ gets bigger, the CKF will be progressively like the traditional KF algorithm. Equations (8), and (9), Equations (26)-(32), and (34) are primarily involved in the CKF algorithm. Remember that the diagonal matrices are $H_{a}(\underline{t})$ and $H_{b}(\underline{t})$, so it is straightforward to get their inverse matrices.

\section{Results and Discussion}

In this article for GPS Receiver position estimation, we proposed a new KF designated as $\mathrm{CKF}$, which utilizes the correntropy criterion as the optimization criterion and applies a fixed-point iterative algorithm to update the posterior estimates. The GPS data required for testing the KF and CKF algorithms is collected from IISc, Bangalore (Lat/Lon: $13.021^{\circ}$ North $/ 77.5^{\circ}$ East) GPS receiver corresponding to $1^{\text {st }}$ January 2018 . The GPS receiver position is estimated using both algorithms (i.e., $\mathrm{KF}$ and $\mathrm{CKF}$ ), and performance analysis is carried out.

Table 1. Estimated receiver position and error in X-direction.

\begin{tabular}{|c|c|c|c|c|}
\hline \multicolumn{3}{|c|}{ Receiver true position in X- direction: 1337936.309m } \\
\hline \multirow{2}{*}{$\begin{array}{c}\text { GPS } \\
\text { Time(Hours) }\end{array}$} & $\begin{array}{c}\text { Receiver estimated position } \\
\text { (meters) }\end{array}$ & \multicolumn{2}{c|}{$\begin{array}{c}\text { Error in position } \\
\text { ( meters) }\end{array}$} \\
\cline { 2 - 5 } & KF & CKF & KF & CKF \\
\hline 9.2881 & 1337902.04 & $\mathbf{1 3 3 7 9 1 2 . 0 9}$ & 34.27 & $\mathbf{2 4 . 2 2}$ \\
\hline 9.2964 & 1337902.04 & $\mathbf{1 3 3 7 9 1 2 . 6 7}$ & 34.27 & $\mathbf{2 3 . 6 4}$ \\
\hline 9.3047 & 1337901.69 & $\mathbf{1 3 3 7 9 1 2 . 6 6}$ & 34.62 & $\mathbf{2 3 . 6 5}$ \\
\hline 9.3131 & 1337902.81 & $\mathbf{1 3 3 7 9 1 2 . 6 6}$ & 33.50 & $\mathbf{2 3 . 6 5}$ \\
\hline 9.3214 & 1337902.81 & $\mathbf{1 3 3 7 9 1 3 . 0 4}$ & 33.50 & $\mathbf{2 3 . 2 7}$ \\
\hline 9.3297 & 1337903.19 & $\mathbf{1 3 3 7 9 1 3 . 9 2}$ & 33.12 & $\mathbf{2 2 . 3 9}$ \\
\hline 9.3381 & 1337903.19 & $\mathbf{1 3 3 7 9 1 3 . 9 9}$ & 33.12 & $\mathbf{2 2 . 3 2}$ \\
\hline 9.3464 & 1337903.71 & $\mathbf{1 3 3 7 9 1 3 . 5 5}$ & 32.60 & $\mathbf{2 2 . 7 6}$ \\
\hline 9.3547 & 1337904.10 & $\mathbf{1 3 3 7 9 1 3 . 8 7}$ & 32.21 & $\mathbf{2 2 . 4 4}$ \\
\hline 9.3631 & 1337904.10 & $\mathbf{1 3 3 7 9 1 2 . 7 8}$ & 32.21 & $\mathbf{2 3 . 5 3}$ \\
\hline
\end{tabular}

A few randomly selected epochs are displayed in the following tables since it is a vast data set. The estimated receiver position and the error in receiver position logged for ten epochs (collected for $30 \mathrm{sec}$ each) in X, Y, Z directions are shown in Tables 1, 2, and 3 . In contrast, the estimated receiver position is shown in Figure 2, and a logged error over 22 hours is shown in Figures 3 and 4, respectively. In addition to the above plots, the smoothened position error (mean position error) over an hour reported with the two algorithms (KF and CKF) is shown in Figures 5 and 6.

Table 2. Estimated receiver position and error in Y-direction.

\begin{tabular}{|c|c|c|c|c|}
\hline \multicolumn{4}{|c|}{ Receiver true position in Y- direction: 6070317.116m } \\
\hline \multirow{2}{*}{$\begin{array}{c}\text { GPS Time } \\
\text { (Hours) }\end{array}$} & $\begin{array}{c}\text { Receiver estimated position } \\
\text { (meters) }\end{array}$ & \multicolumn{2}{c|}{$\begin{array}{c}\text { Error in position } \\
\text { ( meters) }\end{array}$} \\
\cline { 2 - 5 } & KF & CKF & KF & CKF \\
\hline 9.2881 & 6070342.01 & $\mathbf{6 0 7 0 3 3 1 . 5 0}$ & 24.89 & $\mathbf{1 4 . 3 9}$ \\
\hline 9.2964 & 6070342.01 & $\mathbf{6 0 7 0 3 3 1 . 6 3}$ & 24.89 & $\mathbf{1 4 . 5 1}$ \\
\hline 9.3047 & 6070342.27 & $\mathbf{6 0 7 0 3 3 1 . 8 5}$ & 25.15 & $\mathbf{1 4 . 7 4}$ \\
\hline 9.3131 & 6070341.95 & $\mathbf{6 0 7 0 3 3 2 . 3 5}$ & 24.83 & $\mathbf{1 5 . 2 3}$ \\
\hline 9.3214 & 6070341.95 & $\mathbf{6 0 7 0 3 3 1 . 8 3}$ & 24.83 & $\mathbf{1 4 . 7 1}$ \\
\hline 9.3297 & 6070342.44 & $\mathbf{6 0 7 0 3 3 2 . 0 6}$ & 25.32 & $\mathbf{1 4 . 9 4}$ \\
\hline 9.3381 & 6070342.44 & $\mathbf{6 0 7 0 3 3 2 . 0 6}$ & 25.32 & $\mathbf{1 4 . 9 5}$ \\
\hline 9.3464 & 6070341.91 & $\mathbf{6 0 7 0 3 3 2 . 9 4}$ & 24.79 & $\mathbf{1 5 . 8 3}$ \\
\hline 9.3547 & 6070340.90 & $\mathbf{6 0 7 0 3 3 2 . 0 3}$ & 23.79 & $\mathbf{1 4 . 9 2}$ \\
\hline 9.3631 & 6070340.90 & $\mathbf{6 0 7 0 3 3 1 . 9 2}$ & 23.79 & $\mathbf{1 4 . 8 1}$ \\
\hline
\end{tabular}

Table 3. Estimated receiver position and error in Z-direction.

\begin{tabular}{|c|c|c|c|c|}
\hline \multicolumn{3}{|c|}{ Receiver true position in Z- direction: 1427876.908m } \\
\hline \multirow{2}{*}{$\begin{array}{c}\text { GPS Time } \\
\text { (Hours) }\end{array}$} & $\begin{array}{c}\text { Receiver estimated position } \\
\text { (meters) }\end{array}$ & \multicolumn{2}{c|}{$\begin{array}{c}\text { Error in position } \\
\text { ( meters) }\end{array}$} \\
\cline { 2 - 5 } & KF & CKF & KF & CKF \\
\hline 9.2881 & 1427881.32 & $\mathbf{1 4 2 7 8 7 9 . 3 7}$ & 4.42 & $\mathbf{2 . 4 6}$ \\
\hline 9.2964 & 1427881.32 & $\mathbf{1 4 2 7 8 7 9 . 6 6}$ & 4.42 & $\mathbf{2 . 7 5}$ \\
\hline 9.3047 & 1427880.19 & $\mathbf{1 4 2 7 8 7 8 . 8 5}$ & 3.28 & $\mathbf{1 . 9 4}$ \\
\hline 9.3131 & 1427880.04 & $\mathbf{1 4 2 7 8 7 8 . 5 5}$ & 3.14 & $\mathbf{1 . 6 4}$ \\
\hline 9.3214 & 1427880.04 & $\mathbf{1 4 2 7 8 7 9 . 4 2}$ & 3.14 & $\mathbf{2 . 5 1}$ \\
\hline 9.3297 & 1427879.80 & $\mathbf{1 4 2 7 8 7 8 . 7 3}$ & 2.90 & $\mathbf{1 . 8 2}$ \\
\hline 9.3381 & 1427879.80 & $\mathbf{1 4 2 7 8 7 8 . 9 6}$ & 2.90 & $\mathbf{2 . 0 5}$ \\
\hline 9.3464 & 1427879.54 & $\mathbf{1 4 2 7 8 7 8 . 3 4}$ & 2.63 & $\mathbf{1 . 4 3}$ \\
\hline 9.3547 & 1427880.77 & $\mathbf{1 4 2 7 8 7 9 . 4 3}$ & 3.86 & $\mathbf{2 . 5 2}$ \\
\hline 9.3631 & 1427880.77 & $\mathbf{1 4 2 7 8 7 8 . 2 4}$ & 3.86 & $\mathbf{1 . 3 3}$ \\
\hline
\end{tabular}
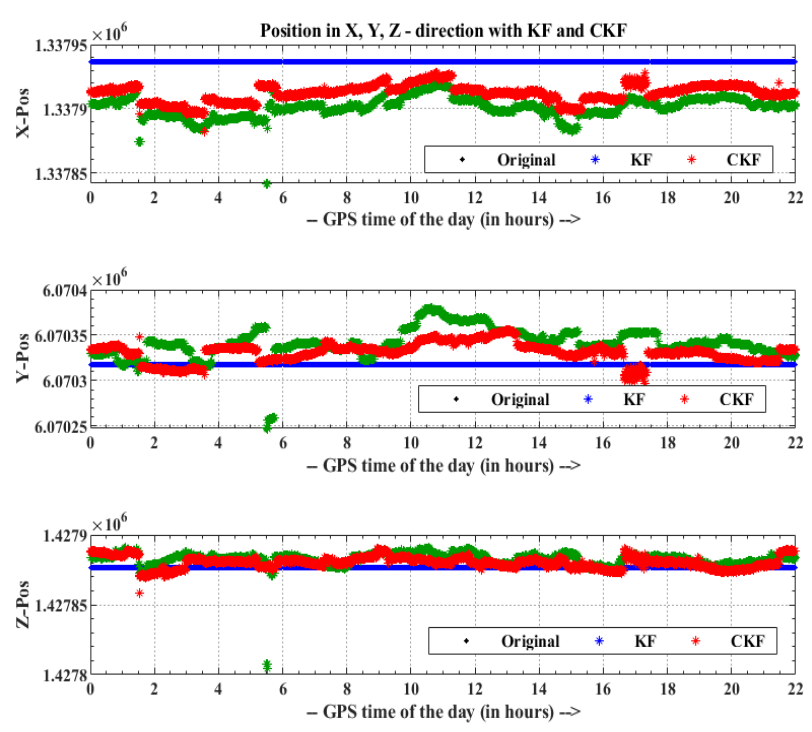

Figure 2. Estimated receiver position vs. time in $\mathrm{X}, \mathrm{Y}, \mathrm{Z}$ - direction using $\mathrm{KF}$ and $\mathrm{CKF}$. 


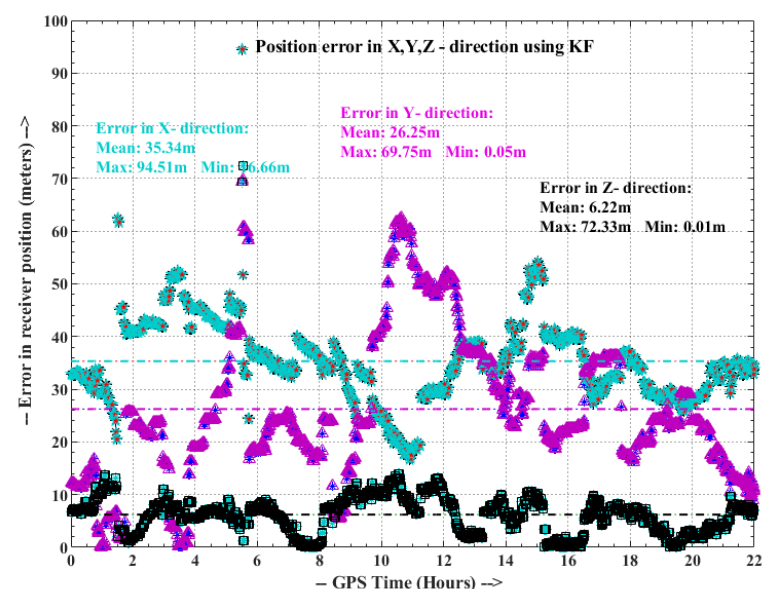

Figure 3. Correlation between time and position error in $\mathrm{X}, \mathrm{Y}, \mathrm{Z}$ direction using KF.

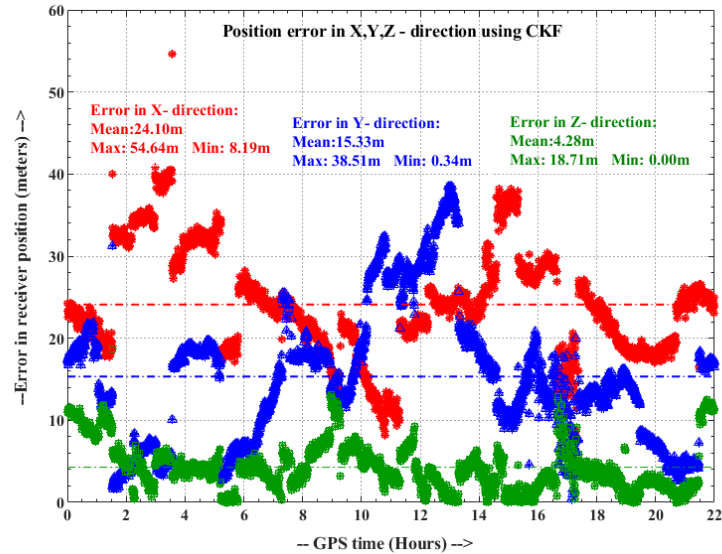

Figure 4. Correlation between time and position error in $\mathrm{X}, \mathrm{Y}, \mathrm{Z}$ direction using CKF.

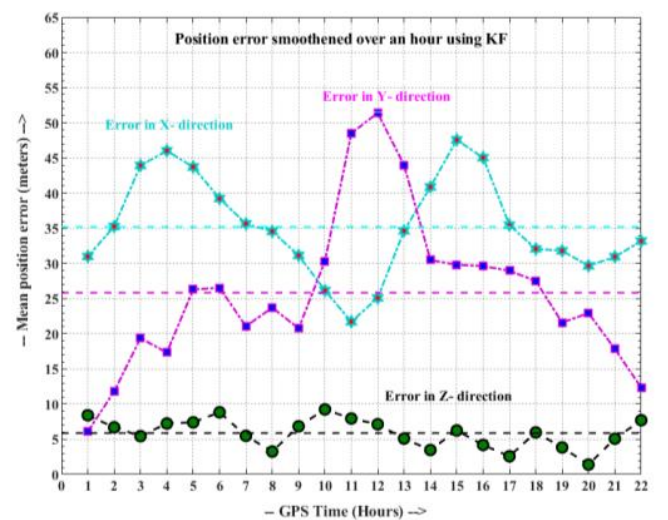

Figure 5. Smoothed position error in X, Y, Z- direction using KF.

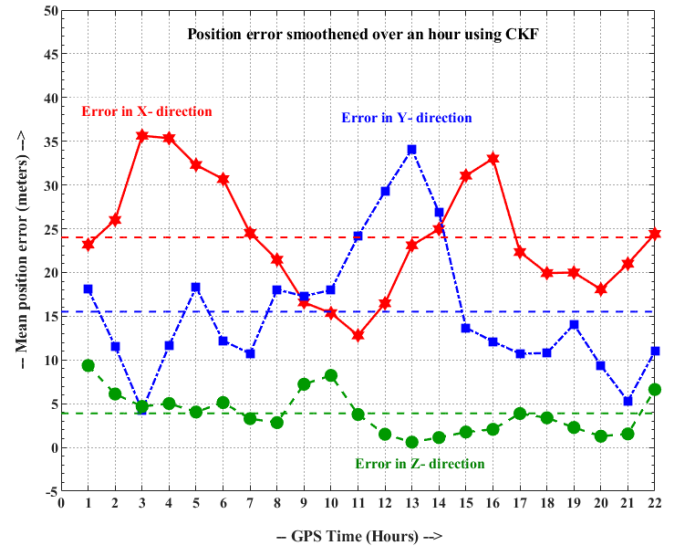

Figure 6. Smoothed position error in X, Y, Z-direction using CKF.

\subsection{Statistical Error Analysis}

Table 4 gives information regarding the three error probability density functions (pdf) obtained by KF and CKF algorithms over IISc, Bangalore GPS receiver data.

Table 4. Descriptive statistics of position error.

\begin{tabular}{|c|c|c|c|c|c|c|}
\hline \multirow{2}{*}{$\begin{array}{c}\text { Error } \\
\text { Scenario }\end{array}$} & \multicolumn{2}{|c|}{$\begin{array}{c}\text { X-direction } \\
\text { (meters) }\end{array}$} & \multicolumn{2}{c|}{$\begin{array}{c}\text { Y-direction } \\
\text { (meters) }\end{array}$} & \multicolumn{2}{c|}{$\begin{array}{c}\text { Z-direction } \\
\text { (meters) }\end{array}$} \\
\cline { 2 - 7 } & KF & CKF & KF & CKF & KF & CKF \\
\hline Mean & 35.34 & $\mathbf{2 4 . 1 0}$ & 26.25 & $\mathbf{1 5 . 3 3}$ & 6.22 & $\mathbf{4 . 2 8}$ \\
\hline Deviation & 7.79 & $\mathbf{6 . 5 4}$ & 13.80 & $\mathbf{8 . 1 7}$ & 4.36 & $\mathbf{2 . 9 3}$ \\
\hline Variance & 60.64 & $\mathbf{4 2 . 7 9}$ & 190.32 & $\mathbf{6 6 . 7 2}$ & 18.97 & $\mathbf{8 . 5 9}$ \\
\hline Minimum & 16.66 & $\mathbf{8 . 1 9}$ & $\mathbf{0 . 0 5}$ & 0.34 & 0.01 & $\mathbf{0 . 0 0}$ \\
\hline Maximum & 94.51 & $\mathbf{5 4 . 6 4}$ & 69.75 & $\mathbf{3 8 . 5 1}$ & 72.33 & $\mathbf{1 8 . 7 1}$ \\
\hline
\end{tabular}

The error analysis parameters are displayed in the above table are calculated for the entire range of data. The performance of the proposed CKF algorithm is compared with that of the traditional $\mathrm{KF}$ algorithm. It is observed from the results that CKF outperforms the KF algorithm by providing high accuracy and low variance position estimation.

The error in estimated position using the $\mathrm{KF}$ and CKF algorithms shown in Figures 3 and 4 are logged over a day (i.e., 22 hours) and are used in the generation of scatter plots. A horizontal scatter plot is a two-dimensional plot; hence only the horizontal position errors ( $\mathrm{X}$ and $\mathrm{Y}$ ) are used in its generation. In contrast, the horizontal and vertical position error scatter plot is a three-dimensional plot that uses the $\mathrm{X}$, $\mathrm{Y}, \mathrm{Z}$ position errors in its generation. Figure 7 and Figure 8 illustrates the comparison of horizontal scatter plot and horizontal and vertical error scatter plot with $\mathrm{KF}$ and $\mathrm{CKF}$ algorithms represented with Circular Error Probable (CEP) and SEP values.

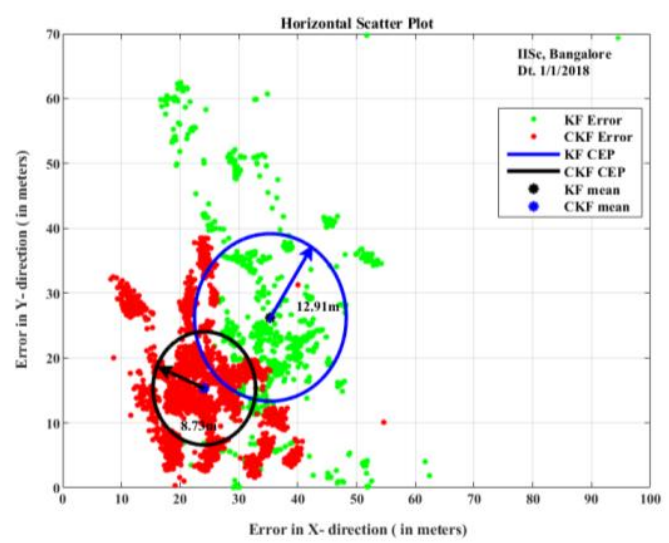

Figure 7. Horizontal position error scatter plot with CEP using KF and CKF.

It is observed from Figures 7 and 8 that the CEP circle and SEP sphere of CKF are nearer to the origin, and the estimated receiver position comes closer to the actual receiver position at $50 \%$ of the time when compared to the KF algorithm. In addition to the above scatter plots, the accuracy metrics of the receiver position (2-D and 3-D) obtained are provided in Table 5. 


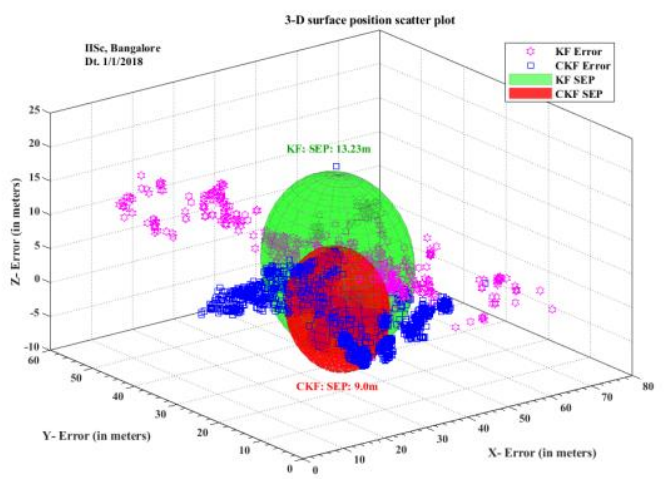

Figure 8. 3-dimensional position error scatter plot with SEP using KF and CKF.

Table 5. Comparison of statistical accuracy measures.

\begin{tabular}{|c|c|c|c|c|c|}
\hline \multicolumn{2}{|c|}{$\begin{array}{l}\text { Statistical } \\
\text { Accuracy } \\
\text { Measure } \\
\text { (SAM) }\end{array}$} & Probability & $\begin{array}{c}\text { KF } \\
\text { (meters) }\end{array}$ & $\begin{array}{c}\text { CKF } \\
\text { (meters) }\end{array}$ & $\begin{array}{l}\text { Improvement in } \\
\text { accuracy with } \\
\text { CKF }(\%)\end{array}$ \\
\hline \multirow{3}{*}{ মি } & DRMS & $65 \%$ & 15.84 & 10.46 & 33.97 \\
\hline & 2DRMS & $95 \%$ & 31.68 & 20.93 & 33.93 \\
\hline & CEP & $50 \%$ & 12.91 & 8.73 & 32.38 \\
\hline \multirow{4}{*}{ ले } & SEP & $50 \%$ & 13.23 & 9.00 & 31.97 \\
\hline & MRSE & $61 \%$ & 16.43 & 10.87 & 33.84 \\
\hline & SAS_90\% & $90 \%$ & 21.61 & 14.69 & 32.02 \\
\hline & SAS_99\% & $99 \%$ & 29.10 & 19.79 & 31.99 \\
\hline
\end{tabular}

It can be observed from Table 5 that $50 \%$ of the estimated horizontal position points (i.e., X, Y) by KF will be within $12.91 \mathrm{~m}$ of the true position, and $50 \%$ of estimated 3-D position points will be within $13.23 \mathrm{~m}$. In contrast, it is $8.73 \mathrm{~m}$ and $9.0 \mathrm{~m}$ for CKF, respectively. However, the CEP and SEP values of CKF show their accuracy better than KF by $4 \mathrm{~m}$ to $5 \mathrm{~m}$. The results show that the proposed method is robust to environmental factors and provides $34 \%$ accuracy improvement over KF.

\section{Conclusions}

A precise navigation algorithm is always essential to provide the user with an accurate position on or above the earth's surface. Therefore, a new navigation algorithm is proposed in this paper, based on the correntropy criterion and the performance of the proposed CKF algorithm is analyzed, and it is found that the proposed CKF navigation algorithm provides the best position estimates when compared to the traditional KF algorithm. Similarly, the values of statistical position accuracy measures are also less for the CKF algorithm than the KF algorithm. For instance, the difference in CEP and SEP values between CKF and $\mathrm{KF}$ is $4.18 \mathrm{~m}$ and $4.23 \mathrm{~m}$, respectively Hence, with due consideration of all correctable errors, it is concluded that the proposed $\mathrm{CKF}$ algorithm provides a significant improvement in position accuracy and helps achieve the accuracy requirement of GPS Aided GEO Augmented Navigation (GAGAN) users, CAT-1 aircraft landings, and other GPS based navigation applications over the low latitude regions.
Table 6. Nomenclature.

\begin{tabular}{|c|c|}
\hline Parameter & Illustration \\
\hline$\sigma$ & Kernel Bandwidth \\
\hline$\varepsilon$ & Small Positive Number \\
\hline$a(\underline{t})$ & State Vector \\
\hline $\mathbf{b}(\mathrm{t})$ & Measurement Vector \\
\hline $\mathbf{Y}$ & $\begin{array}{l}\text { Covariance Matrix Of } \\
\text { Measurement Noise }\end{array}$ \\
\hline $\mathbf{X}$ & $\begin{array}{l}\text { Covariance Matrix Of Process } \\
\text { Noise }\end{array}$ \\
\hline $\mathbf{Z}$ & State Transition Matrix \\
\hline $\mathbf{S}$ & Observation Matrix \\
\hline $\mathbf{Q}$ & Sate Error Covariance Matrix \\
\hline $\mathbf{V}(\underline{\mathrm{t}})$ & Cholesky Factorized Matrix \\
\hline $\mathbf{T}(\underline{\mathrm{t}})$ & Kalman Gain \\
\hline$\hat{\mathbf{a}}(\underline{\mathrm{t}} \mid \mathrm{t}-1)$ & Prior Estimate \\
\hline$\hat{\mathbf{a}}(\underline{\mathrm{t}}-1 \mid \underline{\mathrm{t}}-1)$ & Latest Estimate \\
\hline$\hat{\mathbf{a}}(\underline{\mathrm{t}} \mid \mathrm{t})_{\mathrm{h}}$ & $\begin{array}{c}\text { Updated State Vector at Fixed } \\
\text { Point Iteration } \mathrm{h}=1\end{array}$ \\
\hline $\mathbf{Q}(\underline{\mathrm{t}} \mid \mathrm{t}-1)$ & Prior Covariance Matrix \\
\hline $\mathbf{Q}(\underline{\mathrm{t}} \mid \mathrm{t})$ & Posterior Covariance Matrix \\
\hline $\begin{array}{l}\text { Distance Root } \\
\text { Mean Square } \\
\text { (DRMS) }\end{array}$ & $\sqrt{\operatorname{var}(\mathrm{X})^{2}+\operatorname{var}(\mathrm{Y})^{2}}$ \\
\hline $\begin{array}{l}\text { Twice Distance } \\
\text { Root Mean Square } \\
\text { (2DRMS) }\end{array}$ & $2 * \sqrt{\operatorname{var}(\mathrm{X})^{2}+\operatorname{var}(\mathrm{Y})^{2}}$ \\
\hline $\begin{array}{l}\text { Circular Error } \\
\text { Probable }(\text { CEP })\end{array}$ & $0.62 * \operatorname{var}(\mathrm{Y})+0.56 * \operatorname{var}(\mathrm{X})$ \\
\hline $\begin{array}{l}\text { Spherical Error } \\
\text { Probable (SEP) }\end{array}$ & $0.51 *(\operatorname{var}(\mathrm{X})+\operatorname{var}(\mathrm{Y})+\operatorname{var}(\mathrm{Z}))$ \\
\hline $\begin{array}{l}\text { Mean Radial } \\
\text { Spherical Error } \\
\text { (MRSE) }\end{array}$ & $\sqrt{\operatorname{var}(\mathrm{X})^{2}+\operatorname{var}(\mathrm{Y})^{2}+\operatorname{var}(\mathrm{Z})^{2}}$ \\
\hline $\begin{array}{c}\text { 90\% Spherical } \\
\text { Accuracy Standard } \\
\text { (SAS) }\end{array}$ & $0.833(\operatorname{var}(\mathrm{X})+\operatorname{var}(\mathrm{Y})+\operatorname{var}(\mathrm{Z}))$ \\
\hline $\begin{array}{c}\text { 99\% Spherical } \\
\text { Accuracy Standard } \\
\text { (SAS) }\end{array}$ & $1.122(\operatorname{var}(\mathrm{X})+\operatorname{var}(\mathrm{Y})+\operatorname{var}(\mathrm{Z}))$ \\
\hline
\end{tabular}

The algorithm developed in this paper is validated using the data collected from geographic location IISc, Bangalore, India. Further, by collecting the GPS data from various geographic locations like northern, eastern, western, and southernmost parts, it can be tested and validated for India's Space-Based Augmentation System (SBAS) implementation. The proposed algorithm can be tested during the actual flight trials of CAT-1 and Approach with Vertical (APV) guidance provided by the GAGAN system. It can also be tested for Ground-Based Augmentation System (GBAS) and Local Area Augmentation System (LAAS) that provide the CAT-2 and 3 aircraft landing requirements.

\section{References}

[1] Abdennour S. and Tebbikh H., "Parallel Particle Filters for Multiple Target Tracking," The International Arab Journal of Information Technology, vol. 13, no. 6, pp. 708-715, 2016.

[2] Agarwal R., Meehan M., and O'regan D., Fixed Point Theory and Applications, Cambridge University Press, 2001.

[3] Argyros I. and Hilout S., Computational Methods in Nonlinear Analysis: Efficient Algorithms, 
Fixed Point Theory and Applications, World Scientific, 2013.

[4] Bagadi L., Rao G., and Kumar N., "Firefly, Teaching Learning Based Optimization and Kalman Filter Methods for GPS Receiver Position Estimation," Procedia Computer Science, vol. 143, pp. 892-898, 2018.

[5] Chen B., Liu X., Zhao H., and Principe J., "Maximum Correntropy Kalman Filter," Automatica, vol. 76, pp. 70-77, 2017.

[6] Chen B., Xing L., Liang J., Zheng N., and Principe J., "Steady-State Mean Square Error Analysis for Adaptive Filtering Under the Maximum Correntropy Criterion," IEEE Signal Processing Letters, vol. 21, no. 7, pp. 880-884, 2014.

[7] Choi Min J., Kim Y., Kim E., and Song J., "Enhancement of Heading Accuracy for GPS/INS by Employing Average Velocity in Low Dynamic Situations," IEEE Access, vol. 8, pp. 43826-43837, 2020.

[8] Deep A., Mittal M., and Mittal V., "Application of Kalman Filter in GPS Position Estimation," in Processing of $8^{\text {th }}$ Power India International Conference, Kurukshetra, pp. 1-5, 2018.

[9] Faragher R., "Understanding The Basics of Kalman Filter Via Simple and Intuitive Derivation," IEEE Signal Processing Magazine, vol. 29, no. 5, pp. 128-132, 2012.

[10] Feng X., Feng Y., Zhou F., Ma L., and Yang C., "Nonlinear Non-Gaussian Estimation Using Maximum Correntropy Square Root Cubature Information Filtering," IEEE Access, vol. 8, pp. 181930-181942, 2020.

[11] Julier S., Uhlmann J., and Durrant-Whyte H., "A New Method for the Nonlinear Transformation of Means and Covariances in Filters and Estimators," IEEE Transactions on Automatic Control, vol. 45, no. 3, pp. 477-482, 2000.

[12] Kumar N., Suresh C., and Rao G., "Extended Kalman Filter for GPS Receiver Position Estimation," in Processing of Intelligent Engineering Informatics. Advances in Intelligent Systems and Computing, pp. 481-488, 2018.

[13] Kumar P. and Dutt V., "Navigation Solutions for GPS Receiver Position Estimation over the Southern Region of India," The International Journal of Recent Technology and Engineering, vol. 7, no. 6, pp. 1672-1675, 2019.

[14] Kumar P. and Dutt V., "The Global Positioning System: Popular Accuracy Measures," Materials Today: Proceedings, vol. 33, no. 7, pp. 47974801, 2020.

[15] Li L., Sun Y., and Liu Z., "Maximum Fuzzy Correntropy Kalman Filter and Its Application to Bearings-Only Maneuvering Target Tracking," International Journal of Fuzzy Systems, vol. 23, no. 2, pp. 405-418, 2021.
[16] Liu T., Chen H., Chen Q., Jiang W., Laurichesse D., An X., and Geng T., "Characteristics of Phase Bias from CNES and its Application in MultiFrequency and Multi-GNSS Precise Point Positioning with Ambiguity Resolution," GPS Solutions, vol. 25, no. 2, pp. 1-13, 2021.

[17] Liu W., Pokharel P., and Principe J., "Correntropy: Properties and Applications in Non-Gaussian Signal Processing," IEEE Transactions on Signal Processing, vol. 55, no. 11, pp. 5286-5298, 2007.

[18] Ottacher D., Chan A., Parent E., and Lou E., "Positional and Orientational Accuracy of 3-D Ultrasound Navigation System on Vertebral Phantom Study," IEEE Transactions on Instrumentation and Measurement, vol. 69, no. 9, pp. 6412-6419, 2020.

[19] Oxley A., Uncertainties in GPS Positioning: A Mathematical Discourse, Academic Press, Cambridge, 2017.

[20] Reza Z. and Buehrer M., in Handbook of Position Location: Theory, Practice, and Advances, Wiley-IEEE Press, 2019.

[21] Santra A., Mahato S., Dan S., and Bose A., "Precision of Satellite Based Navigation Position Solution: A Review Using Navic Data," Journal of Information and Optimization Sciences, vol. 40, no. 8, pp. 1683-1691, 2019.

[22] Shokri S., Rahemi N., and Mosavi M., "Improving GPS Positioning Accuracy Using Weighted Kalman Filter and Variance Estimation Methods," CEAS Aeronautical Journal, vol. 11, no. 2, pp. 515-527, 2020.

[23] Tang Z., Sun C., and Liu Z., "The Tracking Algorithm for Maneuvering Target Based on Adaptive Kalman Filter," The International Arab Journal of Information Technology, vol. 10, no. 5, pp. 453-459, 2013.

[24] Zhang Y., Wang J., Sun Q., and Gao W., "Adaptive Cubature Kalman Filter Based on the Variance-Covariance Components Estimation," The Journal of Global Positioning Systems, vol. 15, no. 1, pp. 1-9, 2017. 


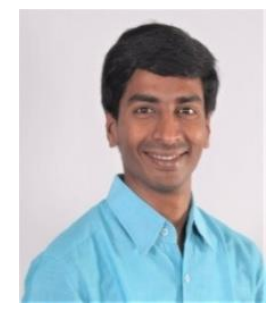

Sirish Kumar Pagoti is currently working as an Assistant Professor in the Department of ECE, Aditya Institute of Technology and Management, Tekkali, India. His areas of research are Navigation Satellite Systems and Satellite Communications. He has 40 publications in various national and international Journals.

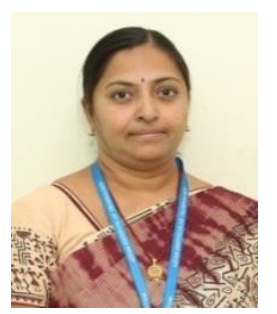

Srilatha Indira Dutt Vemuri is currently working as a Professor in the Department of ECE, GITAM University, Visakhapatnam. Her areas of research are Communication Networks, Global Navigation Satellite Systems, and Satellite Communications. She has more than 90 publications in various national, international conferences and Journals.

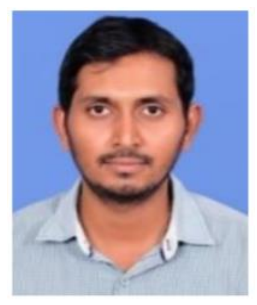

Ganesh Laveti is currently working as an Associate Professor in the Dept. of ECE, GVP College of Engineering for Women, Visakhapatnam. His areas of research are Global Positioning System, Artificial Intelligence and Machine Learning. He has more than 60 publications in various national, international conferences and Journals. 